\title{
A Partial Backlogging Inventory Model for Deteriorating Item under Fuzzy Inflation and Discounting over Random Planning Horizon: A Fuzzy Genetic Algorithm Approach
}

\author{
Dipak Kumar Jana, ${ }^{1}$ Barun Das, ${ }^{2}$ and Tapan Kumar Roy ${ }^{3}$ \\ ${ }^{1}$ Department of Applied Sciences, Haldia Institute of Technology, Haldia, Purba Medinipur 721657, India \\ ${ }^{2}$ Department of Mathematics, Sidho-Kanho-Birsha University, Purulia 723101, India \\ ${ }^{3}$ Department of Mathematics, Bengal Engineering and Science University, Howrah 711103, India \\ Correspondence should be addressed to Dipak Kumar Jana; dipakjana@gmail.com
}

Received 5 December 2012; Accepted 2 June 2013

Academic Editor: Koji Shingyochi

Copyright ( 2013 Dipak Kumar Jana et al. This is an open access article distributed under the Creative Commons Attribution License, which permits unrestricted use, distribution, and reproduction in any medium, provided the original work is properly cited.

\begin{abstract}
An inventory model for deteriorating item is considered in a random planning horizon under inflation and time value money. The model is described in two different environments: random and fuzzy random. The proposed model allows stock-dependent consumption rate and shortages with partial backlogging. In the fuzzy stochastic model, possibility chance constraints are used for defuzzification of imprecise expected total profit. Finally, genetic algorithm (GA) and fuzzy simulation-based genetic algorithm (FSGA) are used to make decisions for the above inventory models. The models are illustrated with some numerical data. Sensitivity analysis on expected profit function is also presented. Scope and Purpose. The traditional inventory model considers the ideal case in which depletion of inventory is caused by a constant demand rate. However, to keep sales higher, the inventory level would need to remain high. Of course, this would also result in higher holding or procurement cost. Also, in many real situations, during a longer-shortage period some of the customers may refuse the management. For instance, for fashionable commodities and hightech products with short product life cycle, the willingness for a customer to wait for backlogging is diminishing with the length of the waiting time. Most of the classical inventory models did not take into account the effects of inflation and time value of money. But in the past, the economic situation of most of the countries has changed to such an extent due to large-scale inflation and consequent sharp decline in the purchasing power of money. So, it has not been possible to ignore the effects of inflation and time value of money any more. The purpose of this paper is to maximize the expected profit in the random planning horizon.
\end{abstract}

\section{Introduction}

In the past few decades, many researches have studied an inventory model with constant demand or dynamic demand (cf. M. K. Maiti and M. Maiti [1], Taleizadeh et al. [2], Jana et al. [3], and others). Moreover, in a competitive situation attractive display of units in the showroom is an important factor. Levin et al. [4] noted that at times the presence of inventory has a motivational effect on the people around it. It is a common belief that large piles of goods displayed in a supermarket will lead the customer to buy more. Thus, many business people use showrooms and the attractive display of units in the showroom to influence the customers. Roy et al.
[5] and Maiti [6] have developed an inventory model with stock-dependent demand.

In most of the earlier inventory models, lifetime of an item is assumed to be infinite while it is in storage. But in reality, many physical goods deteriorate due to dryness, spoilage, vaporization, and so forth and are damaged due to hoarding longer than their normal storage period. The deterioration also depends on preserving facilities and environmental conditions in warehouses/storage. So, due to deterioration effect, a certain fraction of the items either damaged or decayed are not in perfect condition to satisfy the future demand of customers for good items. Deterioration for such items is continuous and constant or time-dependent and/or 
dependent on the on-hand inventory. A number of research papers have already been published on the above type of items by Roy et al. [5] and others.

Moreover, the effects of inflation and time value of money are vital in practical situation, especially in the developing countries with large-scale inflation. Therefore, the effect of inflation and time value of money cannot be ignored in real situations. To relax the assumption of non inflationary effects on costs, Buzacott [7] and Misra [8] simultaneously developed an EOQ model with a constant inflation rate for all associated costs. Bierman and Thomas [9] then proposed an EOQ model under inflation that also incorporated the discount rate. Misra [10] then extended the EOQ model with different inflation rates for various associated costs. Recently, Chern et al. [11] proposed partial backlogging inventory lot-size models for deteriorating items with fluctuating demand under inflation. Maity and Maiti [12] have developed a multiobjective optimal inventory control problem for deteriorating multi-items under fuzzy inflation and discounting. Yang et al. [13] proposed an inventory model under inflation for deteriorating items with stock-dependent consumption rate and partial backlogging shortages.

Use of GA in complex decision making problem is well established Michalewicz [14]. A simple GA starts with a set of potential solutions (called initial population) of the decision making problem under consideration. Individual solutions are called chromosome. Crossover and mutation operations happen among the potential solutions with some probability $p_{c}$ and $p_{m}$ respectively, to get a new set of solutions and it continues until terminating conditions are encountered. Behavior and performance of a GA is directly affected by the interaction between the parameters, that is, selection process of chromosomes for mating pool, $\left(p_{c}, p_{m}\right)$ and so forth. Poor parameter settings usually lead to several problems such as premature convergence. Extensive research work has been made to improve the performance of GA for single/ multiobjective continuous/discrete optimization problems during the last two decades. Michalewicz [14] proposed a genetic algorithm, named contractive mapping genetic algorithm (CMGA), where movement from old population to new population takes place only when average fitness of new population is better than the old one and proved the asymptotic convergence of the algorithm by Banach fixed-point theorem. Bessaou and Siarry [15] proposed a GA where initially more than one population of solutions are generated. Genetic operations are done on every population a finite number of times to find a promising zone of optimum solution. Finally, a population of solutions is generated in this zone and genetic operations are done on this population a finite number of times to get a final solution. Last and Eyal [16] developed a GA with varying population size, where chromosomes are classified into young, middle-aged, and old according to their age and lifetime. Genotype diversity and phenotype diversity of the final population are obtained to measure the performance of the GA. Pezzella et al. [17] developed a GA for the flexible Job-shop scheduling problem, which integrates different strategies for generating the initial population, selecting the individuals for reproduction and reproducing new individuals. In this research paper, an EPQ model of an item is developed in a random planning horizon; that is, lifetime of the product is assumed as random in nature and it follows an exponential distribution with known mean. Unit production cost decreases in each production cycle due to learning effects of the workers on production. Similarly setup cost in each cycle is partly constant and partly decreases in each cycle due to learning effects of the employees. Model is formulated to maximize the expected profit from the whole planning horizon. Following Last and Eyal [16], a GA with varying population size is implemented where chromosomes are classified into young, middle-aged and old according to their age and lifetime. In this GA, crossover probability is a function of parents' age type (young, middle-aged, old, etc.) and is obtained using a fuzzy rule base and fuzzy possibility theory Dubois and Prade [18]. It is an improved GA where a subset of better children is included with the parent population for next generation, and size of this subset is a percentage of the size of its parent set. This GA is used to make optimal decision for the above production inventory model. Performance of the proposed GA for solving the model is compared with that of basic GA and CMGA. The model is illustrated with some numerical data. Sensitivity analysis on expected profit function is also presented. Recently, many papers have been developed in GA (cf. Narmatha Banu and Devaraj [19], Kar et al. [20]).

Due to fuzzy inflation rate, the objective function is fuzzy in nature, and then following Liu and Iwamura [21], M. K. Maiti and M. Maiti [1], the said objective function is converted to a crisp objective function. Some research papers have been already published considering imprecise planning horizon (cf. M. K. Maiti and M. Maiti [1], Roy et al. [5, 22], and others). Till now, none has developed inventory models with both random planning horizon and imprecise effect due to inflation and discounting and stock-dependent demand. In this paper, a partial backlogging inventory model with stockdependent demand for deterioration item has been developed under imprecise inflation rate over a random planning horizon. Here, the planning horizon is stochastic in nature and follows exponential distribution. The expected profit is maximized using a FSGA with roulette wheel selection, arithmetic crossover, and random mutation (Michalewicz, [14]). The models are illustrated with some numerical data. Sensitivity analysis on expected profit is presented.

\section{Assumptions and Notations}

2.1. Assumptions. The mathematical model of the inventory replenishment problem is based on the following assumptions.

(1) Demand rate is assumed to depend on the existing stock level.

(2) The time horizon (a random variable) is finite.

(3) The time horizon completely accommodates first $N$ cycles and ends during $(N+1)$ th cycle.

(4) Lead time is negligible.

(5) Replenishment rate is infinite but replenishment size is finite. 
(6) Shortages are allowed. Unsatisfied demand is partially backlogged. The fraction of shortages backordered is a differentiable and decreasing function of time $t$, denoted by $\delta(t)$, where $t$ is the waiting time up to the next replenishment, and $0 \leq \delta(t) \leq 1$ with $\delta(0)=1$. Note that if $\delta(t)=1$ (or 0 ) for all $t$, then shortages are completely backlogged (or lost).

(7) For deteriorating items, a constant fraction of the on-hand inventory deteriorates per unit of time and there is no repair or replacement of the deteriorated inventory during the planning period.

2.2. Notations. For convenience, the following notations are used throughout the entire paper.

(1) $q(t)$ : on-hand inventory of a cycle at time $t,(j-1) T \leq$ $t \leq j T(j=1,2, \ldots, N)$.

(2) $D(q)$ : the demand rate, where

$$
D(q)= \begin{cases}\alpha+\beta q, & \alpha, \beta \geq 0, q(t) \geq 0, \\ \alpha, & \alpha \geq 0,0 \leq q(t) \leq 0 .\end{cases}
$$

(3) $N$ : number of fully accommodated cycles to be made during the prescribed time horizon.

(4) $T$ : duration of a complete cycle.

(5) $Q_{j}$ : total ordered quantity in $j$ th cycle.

(6) $p$ : selling price per unit.

(7) $C_{3}^{j}: C_{3}+C_{3}^{\prime} e^{-\gamma j}$ is setup cost in $j$ th $(j=1,2, \ldots, N)$ cycle, $\gamma>0 ; C_{3}>0 ; C_{3}^{\prime}>0$. Here $\gamma, C_{3}$, and $C_{3}^{\prime}$ are so chosen to best fit the setup cost function. It is also noted that, set-up cost decreases with the number of cycle due to the learning effect.

(8) $C_{p}$ : the external variable purchasing cost per unit.

(9) $C_{h}$ : the inventory holding cost per unit per unit time.

(10) $C_{b}$ : the backlogging cost per unit per unit time.

(11) $\theta$ : the deterioration rate per unit per unit time.

(12) $r$ : the discount rate.

(13) $k$ : the inflation rate, which is varied by the social economical situations.

(14) $R=r-k$ is the discount rate minus the inflation rate.

(15) $\delta(t)$ : the backlogging rate which is a decreasing function of the waiting time $t$, we here assume that $\delta(t)=e^{-\sigma t}$, where $\sigma>0$, and $t$ is the waiting time.

(16) $\mathrm{HC}_{j}$ : holding cost in the $j$ th cycle.

(17) $\mathrm{HC}_{L}$ : holding cost in the last cycle.

(18) $\mathrm{SR}_{j}$ : sales revenue in the $j$ th cycle.

(19) $\mathrm{SR}_{L}$ : sales revenue in the last cycle.

(20) $\mathrm{PC}_{j}$ : purchasing cost in the $j$ th cycle.

(21) $\mathrm{PC}_{L}$ : purchasing cost in the last cycle.

(22) $\mathrm{TF}(N, T)$ : total profit after completing $N$ fully accommodated cycles.
(23) $\mathrm{TF}_{L}(N, T)$ : total profit for the last cycles.

(24) $E[\mathrm{TF}(T)]$ : expected total profit after completing $N$ fully accommodated cycles.

(25) $E\left[\mathrm{TF}_{L}(T)\right]$ : expected total profit from the last cycle.

(26) $E[\mathrm{TP}(T)]$ : expected total profit from the planning horizon.

(27) $H$ : total time horizon (a random variable) and $h$ is the finite time horizon. Here, it is assumed that the planning horizon $H$ is a random variable and follows exponential distribution with probability density function (p.d.f) as follows:

$$
f(h)= \begin{cases}\lambda e^{-\lambda h}, & h \geq 0 \\ 0, & \text { otherwise }\end{cases}
$$

Here, $\lambda(>0)$ is the parameter of the distribution.

(28) $B(t)$ : the amount of backorders at time $t$.

(29) $Q_{N T}$ : order quantity at time $t=N T$.

\section{Mathematical Formulation}

In the development of the model, we assume that there are $N$ full cycles during the random time horizon $H$ and the planning horizon ends within $(N+1)$ th cycle, that is, within $t=$ $N T$ and $t=(N+1) T$. The $j$ th replenishment is made at time $(j-1) T$. The quantity received at $(j-1) T$ is used partly to meet the accumulated backorders in the previous cycle. The inventory at $(j-1) T$ gradually reduces to zero at $(j-1) T+T^{\prime}$ (cf. Figure 1). For the last cycle, some amount may be left after the end of planning horizon. This amount is sold at a reduced price in a lot.

3.1. Formulation for $j$ th $(1 \leq j \leq N)$ Cycle. The differential equation describing the inventory level $q(t)$ in the interval $(j-1) T \leq t \leq(j-1) T+T^{\prime}(1 \leq j \leq N)$ is given by

$$
\begin{aligned}
& \frac{d q(t)}{d t}=-(\alpha+\beta q(t))-\theta q(t), \\
& (j-1) T \leq t \leq(j-1) T+T^{\prime},
\end{aligned}
$$

where $\alpha, \beta, \theta>0$ and $0<T^{\prime}<T$.

Subject to the conditions that $q(t)=0$ at $t=(j-1) T+T^{\prime}$. The solution of the differential equation (3) is given by

$$
\begin{aligned}
q(t)= & \frac{\alpha}{\beta+\theta}\left(e^{(\beta+\theta)\left((j-1) T+T^{\prime}-t\right)}-1\right), \\
& (j-1) T \leq t \leq(j-1) T+T^{\prime} .
\end{aligned}
$$

During the time interval $\left[(j-1) T+T^{\prime}, j T\right)$, the demand rate $D(q)=\alpha$ and backlogged rate $\sigma(j T-t)=e^{-\sigma(j T-t)}$. Hence, the amount of backorders $B(t)$ is governed by the following differential equation:

$$
\frac{d B(t)}{d t}=-\alpha e^{-\sigma(j T-t)}, \quad(j-1) T+T^{\prime} \leq t \leq j T
$$




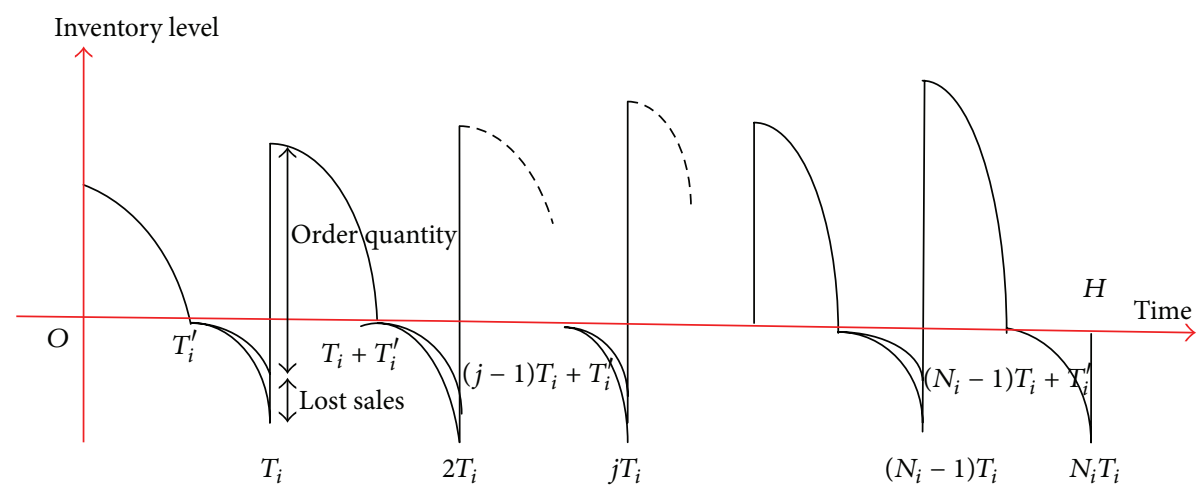

FIgURE 1: Graphical representation of inventory model.

subject to the conditions that $B(t)=0$ at $t=(j-1) T+T^{\prime}$. The solutions of the differential equation (5) is given by

$$
B(t)=\frac{\alpha}{\sigma}\left(e^{-\sigma(j T-t)}-e^{-\sigma\left(T-T^{\prime}\right)}\right), \quad(j-1) T+T^{\prime} \leq t \leq j T .
$$

From (4) and (6), we have the order quantity at $(j-1) T$ in the $j$ th replenishment cycle:

$$
\begin{aligned}
Q_{(j-1) T} & =q((j-1) T)+B((j-1) T) \\
& =\frac{\alpha}{\beta+\theta}\left(e^{(\beta+\theta) T^{\prime}}-1\right)+\frac{\alpha}{\sigma}\left(1-e^{-\sigma\left(T-T^{\prime}\right)}\right) .
\end{aligned}
$$

Present value of holding cost of the inventory for the $j$ th $(1 \leq$ $j \leq N$ ) cycle is given by

$$
\begin{aligned}
\mathrm{HC}_{j}= & C_{h} \int_{(j-1) T}^{(j-1) T+T^{\prime}} q(t) e^{-R t} d t \\
& +C_{b} \int_{(j-1) T+T^{\prime}}^{j T} B(t) e^{-R t} d t \\
= & \frac{\alpha C_{h} e^{-R(j-1) T}}{\beta+\theta}\left\{\frac{e^{(\beta+\theta) T^{\prime}}-e^{-R T^{\prime}}}{\beta+\theta+R}-\frac{1-e^{-R T^{\prime}}}{R}\right\} \\
& +\frac{\alpha C_{b} e^{-R(j-1) T}}{R}\left\{\frac{e^{(R-\sigma)\left(T-T^{\prime}\right)}}{R-\sigma}-\frac{1-e^{-\sigma\left(T-T^{\prime}\right)}}{\sigma}\right\} .
\end{aligned}
$$

Present value of ordering cost for the $j$ th $(1 \leq j \leq N)$ cycle is given by

$$
\begin{aligned}
C_{3}^{j} & =\left(C_{3}+C_{3}^{\prime} e^{-\gamma j}\right) e^{-R(j-1) T}, \quad C_{3}, C_{3}^{\prime}, \gamma>0 \\
& =C_{3} e^{-R(j-1) T}+C_{3} e^{-\gamma} e^{-(\gamma+R T)(j-1)} .
\end{aligned}
$$

Present value of purchasing cost for the $j$ th $(1 \leq j \leq N)$ cycle is given by

$$
\begin{aligned}
\mathrm{PC}_{j}= & C_{p} e^{-R(j-1) T} Q_{(j-1) T} \\
= & C_{p} e^{-R(j-1) T} \\
& \times\left[\frac{\alpha}{\beta+\theta}\left(e^{(\beta+\theta) T^{\prime}}-1\right)+\frac{\alpha}{\sigma}\left(1-e^{-\sigma\left(T-T^{\prime}\right)}\right)\right] .
\end{aligned}
$$

Present value of sales revenue for the $j$ th $(1 \leq j \leq N)$ cycle is given by

$$
\begin{aligned}
\mathrm{SR}_{j}=p[ & e^{-R(j-1) T} B((j-1) T) \\
& \left.+\int_{(j-1) T}^{(j-1) T+t_{1}}\{\alpha+\beta q\} e^{-R t} d t\right] \\
=p[ & \frac{\alpha e^{-R(j-1) T}}{\beta+\theta}\left(e^{(\beta+\theta) T^{\prime}}-1\right) \\
& \left.+\frac{\alpha e^{-R(j-1) T}}{\beta+\theta}\left\{\frac{e^{(\beta+\theta) T^{\prime}}-e^{-R T^{\prime}}}{\beta+\theta+R}-\frac{1-e^{-R T^{\prime}}}{R}\right\}\right] .
\end{aligned}
$$

Then, the present value of the total profit from the $N$ full cycle is as follows:

$$
\begin{aligned}
& \mathrm{TF}(N, T)=\sum_{j=1}^{N}[\left.\mathrm{SR}_{j}-\mathrm{HC}_{j}-C_{3}^{j}-\mathrm{PC}_{j}\right] \\
&=\sum_{j=1}^{N}\left[\frac{p \alpha e^{-R(j-1) T}}{\beta+\theta}\left(e^{(\beta+\theta) T^{\prime}}-1\right)\right. \\
&+\frac{p\left(\alpha-C_{h}\right) e^{-R(j-1) T}}{\beta+\theta} \\
& \times\left\{\frac{e^{(\beta+\theta) T^{\prime}}-e^{-R T^{\prime}}}{\beta+\theta+R}-\frac{1-e^{-R T^{\prime}}}{R}\right\} \\
&-\frac{\alpha C_{b} e^{-R(j-1) T}}{R} \\
& \times\left\{\frac{e^{(R-\sigma)\left(T-T^{\prime}\right)}}{R-\sigma}-\frac{1-e^{-\sigma\left(T-T^{\prime}\right)}}{\sigma}\right\}
\end{aligned}
$$




$$
\begin{array}{r}
-\left[C_{3}+C_{p}\left\{\frac{\alpha}{\beta+\theta}\left(e^{(\beta+\theta) T^{\prime}}-1\right)\right.\right. \\
\left.\left.+\frac{\alpha}{\sigma}\left(1-e^{-\sigma\left(T-T^{\prime}\right)}\right)\right\}\right] \\
\left.\times e^{-R(j-1) T}\right] \\
=\left[\frac{p \alpha}{\beta+\theta}\left(e^{(\beta+\theta) T^{\prime}}-1\right)+\frac{p\left(\alpha-C_{h}\right)}{\beta+\theta}\right. \\
\times\left\{\frac{e^{(\beta+\theta) T^{\prime}}-e^{-R T^{\prime}}}{\beta+\theta+R}-\frac{1-e^{-R T^{\prime}}}{R}\right\} \\
-\frac{\alpha C_{b}}{R}\left\{\frac{e^{(R-\sigma)\left(T-T^{\prime}\right)}}{R-\sigma}-\frac{1-e^{-\sigma\left(T-T^{\prime}\right)}}{\sigma}\right\} \\
-\left[C_{3}+C_{p}\left\{\frac{\alpha}{\beta+\theta}\left(e^{(\beta+\theta) T^{\prime}}-1\right)\right.\right. \\
\left.\left.\left.+\frac{\alpha}{\sigma}\left(1-e^{-\sigma\left(T-T^{\prime}\right)}\right)\right\}\right]\right] \\
\times \sum_{j=1}^{N} e^{-R(j-1) T}+C_{3} e^{-\gamma} \sum_{j=1}^{N} e^{-(\gamma+R T)(j-1)} .
\end{array}
$$

Now,

$$
\sum_{j=1}^{N} e^{-R(j-1) T}=\left(\frac{1-e^{-N R T}}{1-e^{-R T}}\right)
$$

So,

$\operatorname{TF}(N, T)$

$$
\begin{aligned}
= & {\left[\frac{p \alpha}{\beta+\theta}\left(e^{(\beta+\theta) T^{\prime}}-1\right)\right.} \\
& +\frac{p\left(\alpha-C_{h}\right)}{\beta+\theta}\left\{\frac{e^{(\beta+\theta) T^{\prime}}-e^{-R T^{\prime}}}{\beta+\theta+R}-\frac{1-e^{-R T^{\prime}}}{R}\right\} \\
& -\frac{\alpha C_{b}}{R}\left\{\frac{e^{(R-\sigma)\left(T-T^{\prime}\right)}}{R-\sigma}-\frac{1-e^{-\sigma\left(T-T^{\prime}\right)}}{\sigma}\right\} \\
& -\left[C_{3}+C_{p}\left\{\frac{\alpha}{\beta+\theta}\left(e^{(\beta+\theta) T^{\prime}}-1\right)\right.\right. \\
& \left.\left.\left.+\frac{\alpha}{\sigma}\left(1-e^{-\sigma\left(T-T^{\prime}\right)}\right)\right\}\right]\right] \\
& \times\left(\frac{1-e^{-N R T}}{1-e^{-R T}}\right)+C_{3}^{\prime} e^{-\gamma}\left(\frac{1-e^{-N(\gamma+R T)}}{1-e^{-(\gamma+R T)}}\right) .
\end{aligned}
$$

Since the planning horizon $H$ has a p.d.f $f(h)$, the present value of expected total profit from $N$ complete cycles is given by

$$
\begin{aligned}
E[\mathrm{TF}(T)]=\sum_{N=0}^{\infty} & \int_{N T}^{(N+1) T} \operatorname{TF}(N, T) \cdot f(h) d h \\
= & \left\{\left[\frac{p \alpha}{\beta+\theta}\left(e^{(\beta+\theta) T^{\prime}}-1\right)+\frac{p\left(\alpha-C_{h}\right)}{\beta+\theta}\right.\right. \\
& \times\left\{\frac{e^{(\beta+\theta) T^{\prime}}-e^{-R T^{\prime}}}{\beta+\theta+R}-\frac{1-e^{-R T^{\prime}}}{R}\right\} \\
& -\frac{\alpha C_{b}}{R}\left\{\frac{e^{(R-\sigma)\left(T-T^{\prime}\right)}}{R-\sigma}-\frac{1-e^{-\sigma\left(T-T^{\prime}\right)}}{\sigma}\right\} \\
& -\left[C_{3}+C_{p}\left\{\frac{\alpha}{\beta+\theta}\left(e^{(\beta+\theta) T^{\prime}}-1\right)\right.\right. \\
& \times\left(\frac{1-e^{-N R T}}{1-e^{-R T}}\right) \\
& \left.+C_{3}^{\prime} e^{-\gamma}\left(\frac{1-e^{-N(\gamma+R T)}}{1-e^{-(\gamma+R T)}}\right)\right\} \\
\times & \left(e^{-\lambda N T}-e^{-\lambda(N+1) T}\right) .
\end{aligned}
$$

3.2. Formulation for Last Cycle. The differential equations describing the inventory level $q(t)$ in the interval $N T \leq t \leq$ $(N+1) T$ are given by

$$
\begin{gathered}
\frac{d q(t)}{d t}=-\alpha-\beta q-\theta q(t), \quad N T \leq t \leq N T+T^{\prime}, \\
\frac{d B(t)}{d t}=-\alpha e^{-\sigma((N+1) T-t)}, \quad N T+T^{\prime} \leq t \leq(N+1) T,
\end{gathered}
$$

where $\alpha, \beta, \theta>0$, subject to the conditions that

$$
q\left(N T+T^{\prime}\right)=0, \quad B(t)=0 \quad \text { at } t=N T+T^{\prime} .
$$

The solutions of the differential equations (15) and (16) are given by

$$
q(t)=\left\{\begin{array}{c}
\frac{\alpha}{\beta+\theta}\left(e^{(\beta+\theta)\left(N T+T^{\prime}-t\right)}-1\right), \\
N T \leq t \leq N T+T^{\prime}, \\
\frac{\alpha}{\sigma}\left(e^{-\sigma((N+1) T-t)}-e^{-\sigma\left(T-T^{\prime}\right)}\right), \\
N T+t_{1} \leq t \leq(N+1) T+T^{\prime} .
\end{array}\right.
$$




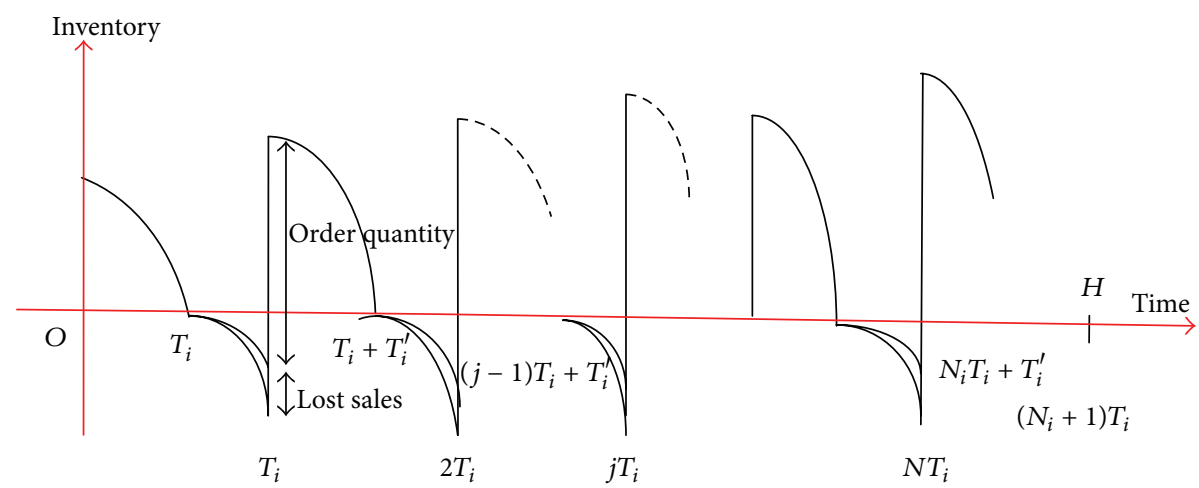

FIGURE 2: Graphical representation of inventory model for Case 1.

From (17), we have the order quantity at NT in the jth replenishment cycle:

$$
\begin{aligned}
Q_{N T} & =q(N T)+B(N T) \\
& =\frac{\alpha}{\beta+\theta}\left(e^{(\beta+\theta) T^{\prime}}-1\right)+\frac{\alpha}{\sigma}\left(e^{-\sigma T}-e^{-\sigma\left(T-T^{\prime}\right)}\right) .
\end{aligned}
$$

Present value of ordering cost is given by

$$
C_{3}^{L}=\left(C_{3}+C_{3}^{\prime} e^{-\gamma(N+1)}\right) e^{-R N T}, \quad C_{3}, C_{3}^{\prime}, \gamma>0 .
$$

Present value of purchasing cost is given by

$$
\begin{aligned}
\mathrm{PC}_{L} & =c Q_{N T} \cdot e^{-R N T} \\
& =c e^{-R N T}\left[\frac{\alpha}{\beta+\theta}\left(e^{(\beta+\theta) T^{\prime}}-1\right)+\frac{\alpha}{\sigma}\left(e^{-\sigma T}-e^{-\sigma\left(T-T^{\prime}\right)}\right)\right] .
\end{aligned}
$$

In last cycle, for simplicity we consider two cases only depending upon the cycle length. Let $h$ be the real value corresponding to the random variable $H$.

Case $1\left(N T<h \leq N T+T^{\prime}\right)$. Present value of holding cost of the inventory for the last cycle (cf. Figure 2 ) is given by

$$
\begin{aligned}
& \mathrm{HC}_{L 1}= C_{h} \int_{N T}^{h} q(t) e^{-R t} d t \\
&= \frac{\alpha C_{h}}{\beta+\theta}\left\{\frac{e^{(\beta+\theta) T^{\prime}-R N T}-e^{(\beta+\theta)\left(N T+N T^{\prime}\right)-(\beta+\theta+R) h}}{\beta+\theta+R}\right. \\
&\left.-\frac{e^{-R N T}-e^{-R h}}{R}\right\} .
\end{aligned}
$$

Present value of sales revenue is given by

$$
\begin{aligned}
\mathrm{SR}_{L 1}= & p \int_{N T}^{h}\{\alpha+\beta q\} e^{-R t} d t \\
= & p\left[\frac{\alpha}{R}\left(e^{-R N T}-e^{-R h}\right)\right. \\
& +\frac{\alpha \beta}{\beta+\theta}\left\{\frac{e^{(\beta+\theta) T^{\prime}-R N T}-e^{(\beta+\theta)\left(N T+T^{\prime}\right)-(\beta+\theta+R) h}}{\beta+\theta+R}\right. \\
- & \left.\left.\frac{\left(e^{-R N T}-e^{-R h}\right)}{R}\right\}\right] .
\end{aligned}
$$

Case $2\left(N T+T^{\prime}<h \leq(N+1) T\right)$. Present value of holding cost of the inventory for the last cycle (Figure 3 ) is given by

$$
\begin{aligned}
\mathrm{HC}_{L 2}= & C_{h} \int_{N T}^{N T+T^{\prime}} q(t) e^{-R t} d t+C_{b} \int_{N T+T^{\prime}}^{h} B(t) e^{-R t} d t \\
= & \frac{\alpha C_{h}}{\beta+\theta}\left\{\frac{e^{(\beta+\theta) T^{\prime}-R N T}-e^{-R\left(N T+T^{\prime}\right)}}{\beta+\theta+R}\right. \\
& \left.-\frac{e^{-R N T}-e^{-R\left(N T+T^{\prime}\right)}}{R}\right\} \\
+\frac{\alpha C_{b}}{\sigma}\left\{\frac{e^{-\sigma\left(T+T^{\prime}\right)-R\left(N T+T^{\prime}\right)}-e^{-\sigma(N+1)-(\sigma+R) h}}{R+\sigma}\right. & \left.-\frac{e^{-\sigma\left(T-T^{\prime}\right)}}{R}\left(e^{-R N T}-e^{-R h}\right)\right\} .
\end{aligned}
$$

Present value of sales revenue is given by

$$
\begin{aligned}
\mathrm{SR}_{L 2}= & p \int_{N T}^{N T+T^{\prime}}\{\alpha+\beta q\} e^{-R t} d t \\
& +p \int_{N T+T^{\prime}}^{h}\left\{\alpha e^{-\sigma((N+1) T-t)}\right\} e^{-R t} d t
\end{aligned}
$$




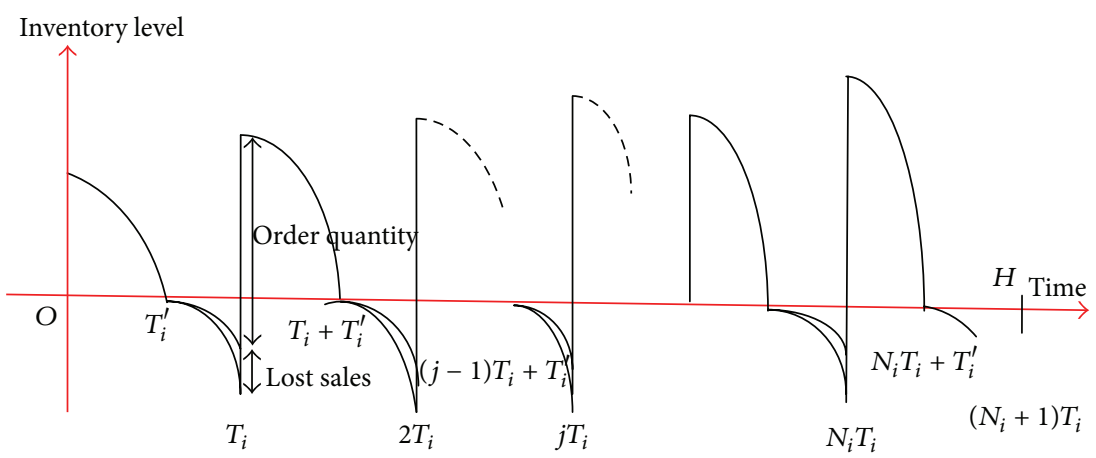

FIGURE 3: Graphical representation of inventory model for Case 2.

$$
\begin{gathered}
=p\left[\alpha T^{\prime}+\frac{\alpha \beta}{\alpha+\beta}\left\{\frac{e^{(\beta+\theta)\left(T^{\prime}+R N T\right)}-e^{-R\left(N T+T^{\prime}\right)}}{\alpha+\beta+R}+T^{\prime}\right\}\right. \\
\left.+\frac{\alpha}{\sigma}\left\{\frac{e^{-\sigma\left(T-T^{\prime}\right)}-e^{-\sigma(N+1) T-(\sigma+R) h}}{\sigma+R}\right\}\right] .
\end{gathered}
$$

So, expected holding cost for the last cycle is given by

$$
\begin{aligned}
& E\left[\mathrm{HC}_{L}\right]=\sum_{N=0}^{\infty} \int_{N T}^{(N+1) T} \mathrm{HC}_{L} \cdot f(h) d h \\
& =\sum_{N=0}^{\infty} \int_{N T}^{N T+t_{1}} \mathrm{HC}_{L 1} \cdot f(h) d h \\
& +\sum_{N=0}^{\infty} \int_{N T+t_{1}}^{(N+1) T} \mathrm{HC}_{L 2} \cdot f(h) d h \\
& =\left[\frac{\alpha C_{h}}{\beta+\theta}\left\{\frac{e^{(\alpha+\beta) T}-e^{-R T^{\prime}}}{\beta+\theta+R}-\frac{1-e^{-\lambda T^{\prime}}}{R}\right\}\right. \\
& \left.+\frac{a C_{b} e^{-\left(T+T^{\prime}+R T\right)}}{\sigma(R+\sigma)}-\frac{e^{-\sigma\left(T+T^{\prime}\right)}}{R}\right] \\
& \times\left(\frac{e^{-\lambda T^{\prime}}-e^{-\lambda T}}{1-e^{-(\lambda+R) T}}\right) \\
& -\left[\frac{\lambda e^{-\sigma T}}{(R+\sigma)(R+\sigma+\lambda)}\right. \\
& \times\left(\frac{e^{-(\lambda+\sigma+R) T^{\prime}}-e^{-(\lambda+\sigma+R) T}}{1-e^{-(\lambda+\sigma+R) T}}\right) \\
& \left.+\frac{e^{-\sigma\left(T+T^{\prime}\right)}}{R(R+\lambda)}\left(\frac{e^{-(R+\lambda) T^{\prime}}-e^{-(R+\lambda) T}}{1-e^{-(R+\lambda) T}}\right)\right] .
\end{aligned}
$$

Expected sales revenue from the last cycle is given by

$$
\begin{aligned}
& E\left[\mathrm{SR}_{L}\right]=\sum_{N=0}^{\infty} \int_{N T}^{(N+1) T} \mathrm{SR}_{L} \cdot f(h) d h \\
& =\sum_{N=0}^{\infty} \int_{N T}^{N T+t_{1}} \mathrm{SR}_{L 1} \cdot f(h) d h \\
& +\sum_{N=0}^{\infty} \int_{N T+t_{1}}^{(N+1) T} \mathrm{SR}_{L 2} \cdot f(h) d h \\
& =p\left[\left\{\frac{\alpha}{R}+\frac{\alpha \beta\left(R e^{(\beta+\theta) T}-1\right)}{R(\theta+\beta)}\right\}\left(\frac{1-e^{\lambda T^{\prime}}}{1-e^{-(\lambda+R) T}}\right)\right. \\
& -\left\{\frac{\lambda \alpha(R \alpha+1)}{R(R+\lambda)}\left(\frac{1-e^{-(\lambda+R) T^{\prime}}}{1-e^{-(\lambda+R) T}}\right)\right. \\
& +\frac{\lambda \alpha \beta e^{\left(\beta+\theta T^{\prime}\right)}}{(\beta+\theta)(\beta+\theta+R)} \\
& \left.\times\left(\frac{1-e^{-(\beta+\theta+\lambda+R) T^{\prime}}}{1-e^{-(\beta+\theta+\lambda+R) T}}\right)\right\} \\
& +\left\{\frac{(\alpha+1) T^{\prime}}{1-e^{-\lambda T}}+\frac{\alpha \beta}{\beta+\theta}\left(\frac{e^{(\beta+\theta) T^{\prime}}-e^{-R T^{\prime}}}{1-e^{-(\lambda+R) T}}\right)\right. \\
& +\frac{\alpha e^{-\sigma\left(T+T^{\prime}\right)}}{\sigma(R+\sigma)}\left(\frac{1-e^{-\lambda T^{\prime}}}{1-e^{-\lambda T}}\right) \\
& \left.\left.-\frac{\lambda e^{-\sigma T}}{(R+\sigma+\lambda)}\left(\frac{1-e^{-(\lambda+R+\sigma) T^{\prime}}}{1-e^{-(\lambda+R) T}}\right)\right\}\right] .
\end{aligned}
$$


Expected reduced selling price from the last cycle is given by

$$
\begin{aligned}
E\left[\mathrm{PC}_{L}\right]= & \sum_{N=0}^{\infty} \int_{N T}^{(N+1) T} \mathrm{PC}_{L} \cdot f(h) d h \\
= & {\left[c \left\{\frac{\alpha}{\beta+\theta}\left(e^{(\beta+\theta) T^{\prime}}-1\right)\right.\right.} \\
& \left.\left.+\frac{\alpha}{\sigma}\left(e^{-\sigma T}-e^{-\sigma\left(T-T^{\prime}\right)}\right)\right\}\right] \\
& \times\left(\frac{1-e^{-\lambda T}}{1-e^{-(\lambda+R) T}}\right) .
\end{aligned}
$$

Expected setup cost from the last cycle is given by

$$
\begin{aligned}
E\left[C_{3}^{L}\right] & =\sum_{N=0}^{\infty} \int_{N T}^{(N+1) T} C_{3}^{L} \cdot f(h) d h \\
& =\left[C_{3}\left(\frac{1-e^{-\lambda T}}{1-e^{-(\lambda+R) T}}\right)+C_{3}^{\prime} e^{-\gamma}\left(\frac{1-e^{-\lambda T}}{1-e^{-(\gamma+\lambda T+R T)}}\right)\right] .
\end{aligned}
$$

So, expected total profit from last cycle is given by

$$
\begin{aligned}
& E\left[\mathrm{TF}_{L}(T)\right] \\
& =E\left[\operatorname{SR}_{L}(T)\right]-E\left[\mathrm{PC}_{L}(T)\right]-E\left[C_{3}^{L}(T)\right]-E\left[\mathrm{HC}_{L}(T)\right] \\
& =p\left[\left\{\frac{\alpha}{R}+\frac{\alpha \beta\left(R e^{(\beta+\theta) T}-1\right)}{R(\theta+\beta)}\right\}\left(\frac{1-e^{\lambda T^{\prime}}}{1-e^{-(\lambda+R) T}}\right)\right. \\
& -\left\{\frac{\lambda \alpha(R \alpha+1)}{R(R+\lambda)}\left(\frac{1-e^{-(\lambda+R) T^{\prime}}}{1-e^{-(\lambda+R) T}}\right)\right. \\
& \left.+\frac{\lambda \alpha \beta e^{\left(\beta+\theta T^{\prime}\right)}}{(\beta+\theta)(\beta+\theta+R)}\left(\frac{1-e^{-(\beta+\theta+\lambda+R) T^{\prime}}}{1-e^{-(\beta+\theta+\lambda+R) T}}\right)\right\} \\
& +\left\{\frac{(\alpha+1) T^{\prime}}{1-e^{-\lambda T}}+\frac{\alpha \beta}{\beta+\theta}\left(\frac{e^{(\beta+\theta) T^{\prime}}-e^{-R T^{\prime}}}{1-e^{-(\lambda+R) T}}\right)\right. \\
& +\frac{\alpha e^{-\sigma\left(T+T^{\prime}\right)}}{\sigma(R+\sigma)}\left(\frac{1-e^{-\lambda T^{\prime}}}{1-e^{-\lambda T}}\right) \\
& \left.\left.-\frac{\lambda e^{-\sigma T}}{(R+\sigma+\lambda)}\left(\frac{1-e^{-(\lambda+R+\sigma) T^{\prime}}}{1-e^{-(\lambda+R) T}}\right)\right\}\right] \\
& -\left[c \left\{\frac{\alpha}{\beta+\theta}\left(e^{(\beta+\theta) T^{\prime}}-1\right)\right.\right. \\
& \left.\left.+\frac{\alpha}{\sigma}\left(e^{-\sigma T}-e^{-\sigma\left(T-T^{\prime}\right)}\right)\right\}+C_{3}\right]
\end{aligned}
$$

$$
\begin{aligned}
& \times\left(\frac{1-e^{-\lambda T}}{1-e^{-(\lambda+R) T}}\right) \\
& -\left[\frac{\alpha C_{h}}{\beta+\theta}\left\{\frac{e^{(\alpha+\beta) T}-e^{-R T^{\prime}}}{\beta+\theta+R}-\frac{1-e^{-\lambda T^{\prime}}}{R}\right\}\right. \\
& \left.\quad+\frac{a C_{b} e^{-\left(T+T^{\prime}+R T\right)}}{\sigma(R+\sigma)}-\frac{e^{-\sigma\left(T+T^{\prime}\right)}}{R}\right] \\
& \times\left(\frac{e^{-\lambda T^{\prime}}-e^{-\lambda T}}{1-e^{-(\lambda+R) T}}\right) \\
& -\left[\frac{\lambda e^{-\sigma T}}{(R+\sigma)(R+\sigma+\lambda)}\left(\frac{e^{-(\lambda+\sigma+R) T^{\prime}}-e^{-(\lambda+\sigma+R) T}}{1-e^{-(\lambda+\sigma+R) T}}\right)\right. \\
& \left.+\frac{e^{-\sigma\left(T+T^{\prime}\right)}}{R(R+\lambda)}\left(\frac{e^{-(R+\lambda) T^{\prime}}-e^{-(R+\lambda) T}}{1-e^{-(R+\lambda) T}}\right)\right] \\
& -C_{3}^{\prime} e^{-\gamma}\left(\frac{1-e^{-\lambda T}}{1-e^{-(\gamma+\lambda T+R T)}}\right) \cdot
\end{aligned}
$$

3.3. Total Profit from the System. Now, total expected profit from the complete time horizon is given by

$$
\begin{aligned}
& E(\mathrm{TP})=E[\mathrm{TF}(T)]+E\left[\mathrm{TF}_{L}(T)\right] \\
& =\left[\frac{p \alpha}{\beta+\theta}\left(e^{(\beta+\theta) T^{\prime}}-1\right)\right. \\
& +\frac{p\left(\alpha-C_{h}\right)}{\beta+\theta}\left\{\frac{e^{(\beta+\theta) T^{\prime}}-e^{-R T^{\prime}}}{\beta+\theta+R}-\frac{1-e^{-R T^{\prime}}}{R}\right\} \\
& -\frac{\alpha C_{b}}{R}\left\{\frac{e^{(R-\sigma)\left(T-T^{\prime}\right)}}{R-\sigma}-\frac{1-e^{-\sigma\left(T-T^{\prime}\right)}}{\sigma}\right\} \\
& -\left[C_{3}+C_{p}\left\{\frac{\alpha}{\beta+\theta}\left(e^{(\beta+\theta) T^{\prime}}-1\right)\right.\right. \\
& \left.\left.\left.+\frac{\alpha}{\sigma}\left(1-e^{-\sigma\left(T-T^{\prime}\right)}\right)\right\}\right]\right] \\
& \times\left(\frac{e^{-\lambda T}-e^{-(R+\lambda) T}}{\left(1-e^{-\lambda T}\right)\left(1-e^{-(R+\lambda) T}\right)}\right) \\
& +p\left[\left\{\frac{\alpha}{R}+\frac{\alpha \beta\left(R e^{(\beta+\theta) T}-1\right)}{R(\theta+\beta)}\right\}\left(\frac{1-e^{\lambda T^{\prime}}}{1-e^{-(\lambda+R) T}}\right)\right. \\
& -\left\{\frac{\lambda \alpha(R \alpha+1)}{R(R+\lambda)}\left(\frac{1-e^{-(\lambda+R) T^{\prime}}}{1-e^{-(\lambda+R) T}}\right)\right. \\
& +\frac{\lambda \alpha \beta e^{\left(\beta+\theta T^{\prime}\right)}}{(\beta+\theta)(\beta+\theta+R)} \\
& \left.\times\left(\frac{1-e^{-(\beta+\theta+\lambda+R) T^{\prime}}}{1-e^{-(\beta+\theta+\lambda+R) T}}\right)\right\}
\end{aligned}
$$




$$
\begin{aligned}
& +\left\{\frac{(\alpha+1) T^{\prime}}{1-e^{-\lambda T}}+\frac{\alpha \beta}{\beta+\theta}\left(\frac{e^{(\beta+\theta) T^{\prime}}-e^{-R T^{\prime}}}{1-e^{-(\lambda+R) T}}\right)\right. \\
& +\frac{\alpha e^{-\sigma\left(T+T^{\prime}\right)}}{\sigma(R+\sigma)}\left(\frac{1-e^{-\lambda T^{\prime}}}{1-e^{-\lambda T}}\right) \\
& \left.\left.-\frac{\lambda e^{-\sigma T}}{(R+\sigma+\lambda)}\left(\frac{1-e^{-(\lambda+R+\sigma) T^{\prime}}}{1-e^{-(\lambda+R) T}}\right)\right\}\right] \\
& -\left[c \left\{\frac{\alpha}{\beta+\theta}\left(e^{(\beta+\theta) T^{\prime}}-1\right)\right.\right. \\
& \left.\left.+\frac{\alpha}{\sigma}\left(e^{-\sigma T}-e^{-\sigma\left(T-T^{\prime}\right)}\right)\right\}+C_{3}\right] \\
& \times\left(\frac{1-e^{-\lambda T}}{1-e^{-(\lambda+R) T}}\right) \\
& -\left[\frac{\alpha C_{h}}{\beta+\theta}\left\{\frac{e^{(\alpha+\beta) T}-e^{-R T^{\prime}}}{\beta+\theta+R}-\frac{1-e^{-\lambda T^{\prime}}}{R}\right\}\right. \\
& \left.+\frac{a C_{b} e^{-\left(T+T^{\prime}+R T\right)}}{\sigma(R+\sigma)}-\frac{e^{-\sigma\left(T+T^{\prime}\right)}}{R}\right] \\
& \times\left(\frac{e^{-\lambda T^{\prime}}-e^{-\lambda T}}{1-e^{-(\lambda+R) T}}\right) \\
& -\left[\frac{\lambda e^{-\sigma T}}{(R+\sigma)(R+\sigma+\lambda)}\right. \\
& \times\left(\frac{e^{-(\lambda+\sigma+R) T^{\prime}}-e^{-(\lambda+\sigma+R) T}}{1-e^{-(\lambda+\sigma+R) T}}\right) \\
& \left.+\frac{e^{-\sigma\left(T+T^{\prime}\right)}}{R(R+\lambda)}\left(\frac{e^{-(R+\lambda) T^{\prime}}-e^{-(R+\lambda) T}}{1-e^{-(R+\lambda) T}}\right)\right] \\
& -C_{3}^{\prime} e^{-\gamma}\left(\frac{1-e^{-\lambda T}}{1-e^{-(\gamma+\lambda T+R T)}}\right) .
\end{aligned}
$$

\section{Models in Different Environments}

4.1. Stochastic Model (Model 1). When the resultant effect of inflation and discounting is crisp in nature, then the present problem is to determine $T$ and $T^{\prime}$ so as to

$$
\max E(\mathrm{TP}) .
$$

4.1.1. Fuzzy Stochastic Model (Model 2). In the real world, deterioration $(\theta)$ and rate of inflation $(R)$ are imprecise in nature, that is, vaguely defined in some situations. So we take $\theta, R$ as fuzzy number, that is, as $\widetilde{\theta}$ and $\widetilde{R}$. Then, due to this assumption, our objective function $E(\mathrm{TP})$ becomes $E(\widetilde{\mathrm{TP}})$. Since optimization of a fuzzy objective is not well defined, so instead of $E(\widetilde{\mathrm{TP}})$ one can optimize its equivalent optimistic or
(1) Set $Z=Z_{0}$.

(2) Generate $R_{0}$ uniformly from the $\alpha_{1}$ cut set of fuzzy number $\widetilde{R}$.

(3) Set $Z_{0}=$ value of $E\{(\mathrm{TP})\}$ for $R=R_{0}$.

(4) If $Z<Z_{0}$ then set $Z=Z_{0}$.

(5) Repeat steps 2, 3 and $4, N$ times, where $N$ is a sufficiently large positive integer.

(6) Return $Z$.

(7) End algorithm.

Algorithm 1: Algorithm to determine $T, T^{\prime}$ and feasible variables for Model 1.

pessimistic return of the objective function. When decision maker likes to optimize the optimistic equivalent of $E(\widetilde{\mathrm{TP}})$, then the problem reduces to the determination of $T, T^{\prime}$ so as to

$$
\begin{array}{ll}
\operatorname{maximize} & Z \\
\text { subject to } & \operatorname{pos}\{E(\widetilde{\mathrm{TP}}) \geq Z\} \geq \alpha_{1} .
\end{array}
$$

Following Liu and Iwamura [21] and others, it can be defuzzified and rewritten as

$$
\begin{array}{ll}
\operatorname{maximize} & Z \\
\text { subject to } & \frac{Z-E\left(\mathrm{TP}_{1}\right)}{E\left(\mathrm{TP}_{2}\right)-E\left(\mathrm{TP}_{1}\right)} \geq \alpha_{1}
\end{array}
$$

(for details see Das et al. [23]).

\section{Solution Procedure}

To solve the stochastic model 1, GA is used. The basic technique to deal with problems (35) is to convert the possibility constraint to its deterministic equivalent. However, the procedure is usually very hard and successful in some particular cases. Following Liu and Iwamura [21], M. K. Maiti and M. Maiti [1], here two simulation algorithms are proposed to determine $Z$ in (35), for a feasible $T$.

To determine $T, T^{\prime}$ for feasible variables, roughly find a point $R_{0}$ from fuzzy number $\widetilde{R}$, which approximately minimizes $Z$. Let this value be $Z_{0}$ and set $Z=Z_{0}$. (For simplicity one can take $Z_{0}=0$.) Then, $p_{0}$ is randomly generated in $\alpha_{1}$ cut set of $\widetilde{R}$ and let $Z_{0}=$ value of $\widetilde{E(\mathrm{TP})}$ for $R=R_{0}$ and if $Z<$ $Z_{0}$ replace $Z$ with $Z_{0}$. This step is repeated a finite number of times and final value is taken as value of $Z$. This phenomenon is used to develop Algorithm 1.

Now roughly find a point $R$ from fuzzy number $\widetilde{R}$, which approximately minimizes $E\{(\mathrm{TP})\}$. Let this value be $Z_{0}$ (for simplicity one can take $Z_{0}=0$ also) and $\varepsilon$ a positive number. Set $Z=Z_{0}-\varepsilon$ and if $\operatorname{pos}\{E(\widetilde{\mathrm{TP}})<Z\} \leq 1-\alpha_{2}$, then increase $Z$ with $\varepsilon$. Again check pos $\{E(\widetilde{\mathrm{TP}})<Z\} \leq 1-\alpha_{2}$ and it continues until $\operatorname{pos}\{E(\widetilde{\mathrm{TP}})<Z\}>1-\alpha_{2}$. At this stage, decrease the value of $\varepsilon$ and again try to improve $Z$. When $\varepsilon$ becomes sufficiently, small, then we stop, and the final value of $Z$ is taken 
(1) Set $Z=Z_{0}-\varepsilon, F=Z_{0}-\varepsilon, F_{0}=Z_{0}-\varepsilon$, tol $=0.0001$.

(2) Generate $p_{0}$ uniformly from the $1-\alpha_{2}$ cut set of fuzzy number $\widetilde{p}$.

(3) Set $Z_{0}=$ value of $E\left\{f(x, y, p)\right.$ for $p=p_{0}$.

(4) If $Z_{0}<Z$.

(5) then go to step 11.

(6) End If

(7) Repeat step-2 to step- $6 N$ times.

(8) Set $F=Z$.

(9) Set $Z=Z+\varepsilon$.

(10) Go to step-2.

(11) If $(Z=F) / /$ In this case optimum value of $Z<Z_{0}-\varepsilon$

(12) Set $Z=F_{0}-\varepsilon, F=F-\varepsilon, F_{0}=F_{0}-\varepsilon$.

(13) Go to step-2

(14) End If

(15) If $(\varepsilon<t o l)$

(16) go to step-21

(17) End If

(18) $\varepsilon=\varepsilon / N$

(19) $Z=F+\varepsilon$

(20) Go to step-2.

(21) Output $F$.

Algorithm 2: An algorithm to determine $Z$, for Model 2.

(1) $T \leftarrow 0$.

(2) Initialize $p_{c}, p_{m}$.

(3) Initialize $(P(T))$ and let $N$ be its size.

(4) Evaluate $(P(T))$.

(5) While (Not termination condition)

(6) Select $N$ solutions from $P(T)$ for mating pool using roulette-wheel selection process (one solution may be selected more than once). Let this set be $P_{1}(T)$.

(7) Select solutions from $P_{1}(T)$ for crossover depending on $p_{c}$.

(8) Perform crossover on selected solutions to obtain population $P_{1}(T)$.

(9) Select solutions from $P_{1}(T)$ for mutation depending on $p_{m}$.

(10) Perform mutation on selected solutions to obtain new population $P(T+1)$.

(11) Evaluate $(P(T+1))$.

(12) If average fitness of $P(T+1)>$ average fitness of $P(T)$ then

(13) $\quad T \leftarrow T+1$.

(14) End If.

(15) End While.

(16) Output: Best solution of $P(T)$.

(17) End algorithm.

Algorithm 3: FSGA algorithm.

as value of $Z$. Using this criterion, required algorithm is developed as shown in Algorithm 2. In this algorithm, the variable $F_{0}$ is used to store initial assumed value of $Z$ and $F$ is used to store the value of $Z$ in each iteration.

Therefore for feasible value of the variables, we determine $Z$ using the above algorithms and to optimize $Z$ we use GA.
Since fuzzy simulation algorithm is used to determine $Z$ in the algorithm, this GA is named a fuzzy simulation based genetic algorithm (FSGA). This algorithm is named FSGA when fuzzy simulation process is used to determine objective function value.

5.1. Fuzzy Simulation Based Single Objective Genetic Algorithm (FSGA). In natural genesis, we know that chromosomes are the main carriers of the hereditary information from parents to offsprings and that genes, which present hereditary factors, are lined up in chromosomes. At the time of reproduction, crossover and mutation take place among the chromosomes of parents. In this way, hereditary factors of parents are mixed up and carried over to their offsprings. Darwinian principle states that only the fittest animals can survive in nature. So a pair of fittest parents normally reproduce better offspring.

The above-mentioned phenomenon is followed to create a genetic algorithm for an optimization problem. Here potential solutions of the problem are analogous with the chromosomes and chromosome of better offspring with the better solution of the problem. Crossover and mutation occur among a set of potential solutions and obtained a new set of solutions and it continues until terminating conditions are encountered. Michalewicz [14] proposed a genetic algorithm named the contractive mapping genetic algorithm (CMGA) and proved the asymptotic convergence of the algorithm by the Banach fixed-point theorem. In CMGA, movement from an old population to a new population takes place only when the average fitness of a new population is better than the old one. This algorithm is modified with the help of a fuzzy simulation process to solve the model in some cases. The algorithm is named FSGA and this is presented below. In this algorithm, $p_{c}, p_{m}$ are probabilities of the crossover and the probability of mutation, respectively, $T$ is the iteration counter, and $P(T)$ is the population of potential solutions for iteration $T$. The initialize $(P(T))$ function initializes the population $P(T)$ at the time of initialization. The evaluate $(P(T))$ function evaluates the fitness of each member of $P(T)$, and at this stage an objective function value due to each solution is evaluated via the fuzzy simulation process (using Algorithm 1).

\subsubsection{FSGA Algorithm. See Algorithm 3.}

\subsubsection{FSGA Procedures}

(a) Representation: An " $n$-dimensional real vector" $X=$ $\left(x_{1}, x_{2}, \ldots, x_{n}\right)$ is used to represent a solution, where $x_{1}, x_{2}, \ldots, x_{n}$ represent $n$ decision variables of the problem.

(b) Initialization: $N$ such solutions $X_{1}, X_{2}, X_{3}, \ldots, X_{N}$ are randomly generated by random number generator such that each $X_{i}$ satisfies the constraints of the problem. Constraints of the problem are satisfied using Algorithm 1. This solution set is taken as initial population $P(1)$. Also set $p_{c}=0.3, p_{m}=0.2$, and $T=1$.

(c) Fitness value: value of the objective function due to the solution $X$ is taken as fitness of $X$. Let it be $f(X)$. 
TABLE 1: Results for stochastic Model 1.

\begin{tabular}{cccccc}
\hline$\alpha$ & $\beta$ & $T$ & $T^{\prime}$ & $Q$ & $E(\mathrm{TP})$ \\
\hline \multirow{3}{*}{50} & 0.75 & 1.686 & 1.208 & 50.005 & 12993.57 \\
& 0.70 & 1.692 & 1.214 & 46.894 & 11846.67 \\
& 0.65 & 1.703 & 1.229 & 44.564 & 11268.40 \\
\hline \multirow{4}{*}{45} & 0.75 & 1.693 & 1.228 & 46.700 & 12473.83 \\
& 0.70 & 1.712 & 1.247 & 45.830 & 11372.80 \\
& 0.65 & 1.743 & 1.290 & 44.689 & 10817.66 \\
\hline \multirow{4}{*}{40} & 0.75 & 1.746 & 1.287 & 46.306 & 11694.21 \\
& 0.70 & 1.792 & 1.298 & 43.755 & 10662.00 \\
& 0.65 & 1.804 & 1.302 & 40.558 & 10141.56 \\
\hline
\end{tabular}

TABLE 2: Value of $E(T P)$ for different optimistic level for Model 2.

\begin{tabular}{lcccccc}
\hline$\alpha_{1}$ & 0.0 & 0.2 & 0.4 & 0.6 & 0.8 & 1.0 \\
\hline$E(\mathrm{TP})$ & 11748.45 & 11993.73 & 12102.19 & 12404.38 & 12884.38 & 12902.58 \\
\hline
\end{tabular}

TABLE 3: Results for fuzzy stochastic model.

\begin{tabular}{lcc}
\hline$\beta$ & $\theta$ & $\begin{array}{c}\text { Optimistic return } E(\mathrm{TP}) \\
\left(\alpha_{1}=0.9\right)\end{array}$ \\
\hline 0.00 & 0.100 & 11748.43 \\
0.50 & 0.100 & 11859.25 \\
0.75 & 0.100 & 11993.25 \\
0.00 & 0.125 & 11895.29 \\
0.50 & 0.125 & 12007.49 \\
0.75 & 0.125 & 12143.60 \\
0.00 & 0.150 & 12638.74 \\
0.50 & 0.150 & 12757.96 \\
0.75 & 0.150 & 12902.58 \\
\hline
\end{tabular}

Objective function is evaluated via fuzzy simulation process (using Algorithm 1 or Algorithm 2).

(d) Selection process for mating pool: the following steps are followed for this purpose.

(i) Find total fitness of the population $F=$ $\sum_{i=1}^{N} f\left(X_{i}\right)$.

(ii) Calculate the probability of selection $p_{i}$ of each solution $X_{i}$ by the formula $p_{i}=f\left(X_{i}\right) / F$.

(iii) Calculate the cumulative probability $q_{i}$ for each solution $X_{i}$ by the formula $q_{i}=\sum_{j=1}^{i} p_{j}$.

(iv) Generate a random number " $r$ " from the range $[0,1]$.

(v) If $r<q_{1}$, then select $X_{1}$ otherwise select $X_{i}(2 \leq$ $i \leq N$ ) where $q_{i}-1 \leq r \leq q_{i}$.

(vi) Repeat step, (iv) and (v) $N$ times to select $N$ solutions from old population. Clearly, one solution may be selected more than once.

(vii) Selected solution set is denoted by $P_{1}(T)$ in the proposed FSGA algorithm. (e) Crossover

(i) Selection for crossover: for each solution of $P(T)$ generate a random number $r$ from the range $[0,1]$. If $r<p_{c}$, then the solution is taken for crossover, where $p_{c}$ is the probability of crossover.

(ii) Crossover process: crossover took place on the selected solutions. For each pair of coupled solutions $Y_{1}, Y_{2}$, a random number $c$ is generated from the range $[0,1]$, and their offsprings $Y_{11}$ and $Y_{21}$ are obtained by the formula:

$$
\begin{aligned}
& Y_{11}=c Y_{1}+(1-c) Y_{2}, \\
& Y_{21}=c Y_{2}+(1-c) Y_{1} .
\end{aligned}
$$

\section{(f) Mutation}

(i) Selection for mutation: for each solution of $P(T)$ generate a random number $r$ from the range $[0,1]$. If $r<p_{m}$, then the solution is taken for mutation, where $p_{m}$ is the probability of mutation.

(ii) Mutation process: to mutate a solution $X=$ $\left(x_{1}, x_{2}, \ldots, x_{n}\right)$ select a random integer $r$ in the range $[1, n]$. Then, replace $x_{r}$ by randomly generated value within the boundary of $r$ th component of $X$.

\section{Numerical Illustration}

To solve the stochastic model (Model 1), genetic algorithm (GA) (Section 5.1) is used and fuzzy stochastic model (Model 2) is solved by fuzzy simulation based genetic algorithm (FSGA) (Section 6). The corresponding parameters in GA and FSGA are POPSIZE $=50, \mathrm{PCROS}=0.2$, $\mathrm{PMUTE}=0.2$, and $M A X G E N=50$. A real-number presentation is used here. In this representation, each chromosome $\mathrm{X}$ is a string of $m$ (here, $m=1$ ) number of genes, these represent decision variables. For each chromosome X, every gene, which represents the independent variables (here, $T$ ), is randomly generated between its boundaries until it is feasible. In this problem, arithmetic crossover and random mutation are applied to generate new offsprings.

To illustrate the models, we consider the following numerical data: $p=100, C_{3}=50, C_{3}^{\prime}=40, C_{h}=15, C_{p}=3$, $C_{b}=5, \gamma=0.05, \theta=0.1,80, \sigma=0.08, \lambda=0.01, r=0.25$, and $i=0.05$; that is, $R=0.2$ in appropriate units.

6.1. Stochastic Model 1. The optimal values of $T$ along with maximum expected total profit have been calculated for different values of $\alpha$ and $\beta$; results are displayed in Table 1 .

It is observed that as $\alpha$ and $\beta$ increase, expected profit increases due to the increase of demand henceforth selling amount. Moreover for increasing values of $\alpha$ and $\beta$, as length of business periods decreases, average expected profit increases. All these observations agree with reality. 
TABLE 4: Sensitivity analysis with respect to present inflation rate for stochastic model.

\begin{tabular}{|c|c|c|c|c|c|}
\hline$R$ & Percentage change in $R$ & $\begin{array}{l}\text { Percentage change in } \\
\text { expected total profit } \\
(\theta=0.1 \text { and } \beta=3.5)\end{array}$ & $\begin{array}{l}\text { Percentage change in } \\
\text { expected total profit } \\
(\theta=0.15 \text { and } \beta=3.5)\end{array}$ & $\begin{array}{l}\text { Percentage change in } \\
\text { expected total profit } \\
(\theta=0.1 \text { and } \beta=4.0)\end{array}$ & $\begin{array}{c}\text { Percentage change in } \\
\text { expected total profit } \\
(\theta=0.15 \text { and } \beta=4.0)\end{array}$ \\
\hline 0.06 & -40 & +75.80 & +75.80 & +65.05 & +65.86 \\
\hline 0.07 & -30 & +42.75 & +42.63 & +42.67 & +42.54 \\
\hline 0.08 & -20 & +26.14 & +26.07 & +25.09 & +26.01 \\
\hline 0.09 & -10 & +12.41 & +12.59 & +11.22 & +09.96 \\
\hline 0.10 & 00 & $\begin{array}{c}0.0 \\
\left({ }^{*} 12993.57\right)\end{array}$ & $\begin{array}{c}0.0 \\
\left({ }^{*} 12884.38\right)\end{array}$ & $\begin{array}{c}0.0 \\
\left({ }^{*} 12102.19\right)\end{array}$ & $\begin{array}{c}0.0 \\
\left({ }^{*} 11993.73\right)\end{array}$ \\
\hline 0.11 & +10 & -09.27 & -08.26 & -09.25 & -07.52 \\
\hline 0.12 & +20 & -17.07 & -17.03 & -15.57 & -16.98 \\
\hline 0.13 & +30 & -23.69 & -23.64 & -23.63 & -23.58 \\
\hline 0.14 & +40 & -30.52 & -30.563 & -30.56 & -30.20 \\
\hline
\end{tabular}

* Indicate original value, others show the values in percentage.

TABLE 5: Sensitivity analysis with respect to the parameter $\lambda$ for stochastic model.

\begin{tabular}{|c|c|c|c|c|c|}
\hline$\lambda$ & Percentage change in $\lambda$ & $\begin{array}{l}\text { Percentage change in } \\
\text { expected total profit } \\
(\theta=0.1 \text { and } \beta=3.5)\end{array}$ & $\begin{array}{l}\text { Percentage change in } \\
\text { expected total profit } \\
(\theta=0.15 \text { and } \beta=3.5)\end{array}$ & $\begin{array}{l}\text { Percentage change in } \\
\text { expected total profit } \\
(\theta=0.1 \text { and } \beta=4.0)\end{array}$ & $\begin{array}{l}\text { Percentage change in } \\
\text { expected total profit } \\
(\theta=0.15 \text { and } \beta=4.0)\end{array}$ \\
\hline 0.030 & -40 & +21.56 & +21.53 & +21.52 & +21.24 \\
\hline 0.035 & -30 & +14.44 & +14.60 & +14.52 & +14.63 \\
\hline 0.040 & -20 & +09.62 & +10.03 & +09.63 & +09.71 \\
\hline 0.045 & -10 & +04.83 & +04.82 & +04.79 & +04.83 \\
\hline 0.050 & 00 & $\begin{array}{c}0.0 \\
\left({ }^{*} 12933.69\right)\end{array}$ & $\begin{array}{c}0.0 \\
\left({ }^{*} 12884.23\right)\end{array}$ & $\begin{array}{c}0.0 \\
\left({ }^{*} 12102.29\right)\end{array}$ & $\begin{array}{c}0.0 \\
\left({ }^{*} 11992.96\right)\end{array}$ \\
\hline 0.055 & +10 & -04.72 & -04.78 & -04.74 & -4.78 \\
\hline 0.059 & +20 & -09.58 & -09.51 & -09.45 & -09.52 \\
\hline 0.065 & +30 & -14.06 & -14.20 & -14.10 & -14.22 \\
\hline 0.070 & +40 & -19.23 & -19.23 & -19.25 & -19.25 \\
\hline
\end{tabular}

${ }^{*}$ Indicate original value, others show the values in percentage.

6.2. Fuzzy Stochastic Model 2. Here, the resultant inflationary effect and deterioration rate are considered as a triangular fuzzy number; that is, $\widetilde{R}=\widetilde{r}-\widetilde{k}=(0.19,0.25,0.31)-(0.04$, $0.05,0.06)=(0.15,0.2,0.25)$ and $\tilde{\theta}=(0.08,0.1,0.12)$ for $\alpha=$ $50, \beta=0.75$, and all other data remain the same as in stochastic model. The maximum optimistic returns have been calculated for different levels of optimistic.

From Table 2, it is revealed that as the possibility level increases, total expected profit increases as expected. And it is also shown that expected total profit for $\alpha=1.0$ is the same in Table 3.

6.3. Sensitivity Analysis. A sensitivity analysis is performed for stochastic model with respect to different resultant inflationary effect $(R)$ for crisp inflation, and results are presented in Table 4. It is observed that as $R$ increases profit decreases which agrees with reality.

A sensitivity analysis is performed for the maximum expected total profit with respect to the different values of parameter $\lambda$ for stochastic model and is presented in Table 5 .
It is observed that as $\lambda$ decreases profit increases. This happens because as $\lambda$ decreases expected time horizon increases which increases the total expected profit.

Results due to different values of confidence levels $\alpha_{1}$ for Model 2 is calculated and depicted in Figure 2. In both cases, as expected, profit decreases with the increase of confidence levels. The graphical representation of possibility threshold versus expected profit is depicted in Figure 4.

\section{Concluding Remarks}

In this paper, a realistic stock-dependent inventory model with shortages and partial backlogging has been formulated with fuzzy deterioration, inflation rate, and setup cost with learning effect in random planning horizon. Until now, no inventory model has been formulated in such consideration, that is, stock-dependent demand, shortages with partial backlogging, effect of inflation, learning effect, deterioration, random planning horizon, and so forth, in both stochastic and fuzzy stochastic environments. The proposed models are optimized via soft computing methods: GA and FSGA. The 


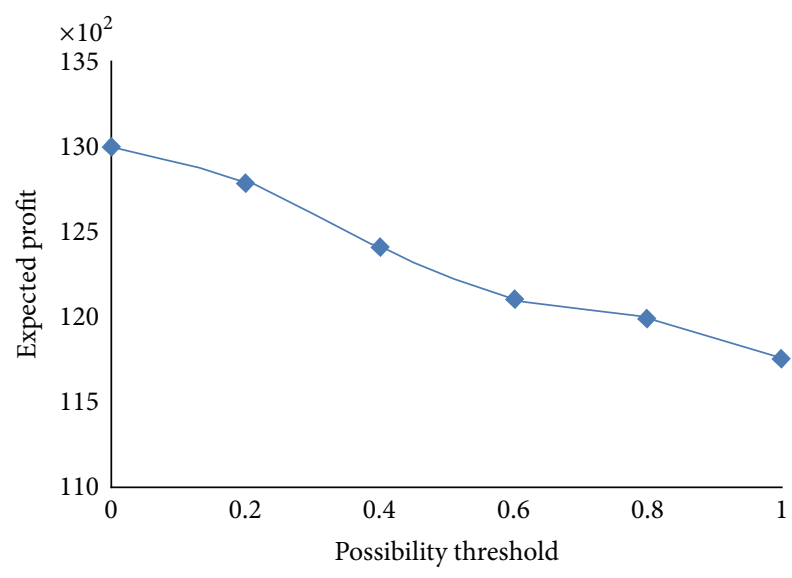

Figure 4: Possibility threshold versus expected profit.

present concept can be extended to production planning model, multi-item production planning model, optimal control problem for multiproduct manufacturing also, and so forth, which may be areas of future research.

\section{Acknowledgments}

The authors are very grateful to the respected editors of this journal for improvement of the paper. This work is supported by the University Grants Commission, India, vide F.PSW103/10-11 (ERO) dated 20.10.10.

\section{References}

[1] M. K. Maiti and M. Maiti, "Fuzzy inventory model with two warehouses under possibility constraints," Fuzzy Sets and Systems, vol. 157, no. 1, pp. 52-73, 2006.

[2] A. A. Taleizadeh, S. T. A. Niaki, and R. Nikousokhan, "Constraint multiproduct joint-replenishment inventory control problem using uncertain programming," Applied Soft Computing Journal, vol. 11, no. 8, pp. 5143-5154, 2011.

[3] D. K. Jana, K. Maity, and T. K. Roy, "A bi-fuzzy approach to a production-recycling-disposal inventory problem with environment pollution cost via genetic algorithm," International Journal of Computer Applications, vol. 61, pp. 1-10, 2013.

[4] R. I. Levin, C. P. Mcaughlim, P. R. Lamone, and J. F. Kottas, Production Management/ Operations Management: (Contemporary Policy for Managing Operating System), McGraw-Hill, New York, NY, USA, 1972.

[5] A. Roy, M. K. Maiti, S. Kar, and M. Maiti, “Two storage inventory model with fuzzy deterioration over a random planning horizon," Mathematical and Computer Modelling, vol. 46, no. 1112, pp. 1419-1433, 2007.

[6] M. K. Maiti, "A fuzzy genetic algorithm with varying population size to solve an inventory model with credit-linked promotional demand in an imprecise planning horizon," European Journal of Operational Research, vol. 213, no. 1, pp. 96-106, 2011.

[7] J. A. Buzacott, "Economic order quantities with inflation," Operational Research Quarterly, vol. 26, no. 3, pp. 553-558, 1975.

[8] R. B. Misra, "A study of inflationary effects on inventory systems," Logistic Spectrum, vol. 9, pp. 260-268, 1975.
[9] H. Bierman and J. Thomas, "Inventory decisions under inflationary conditions," Decision Sciences, vol. 8, pp. 151-155, 1997.

[10] R. B. Misra, "Note on optimal inventory management under inflation," Naval Research Logistics Quarterly, vol. 26, no. 1, pp. 161-165, 1979.

[11] M.-S. Chern, H.-L. Yang, J.-T. Teng, and S. Papachristos, "Partial backlogging inventory lot-size models for deteriorating items with fluctuating demand under inflation," European Journal of Operational Research, vol. 191, no. 1, pp. 127-141, 2008.

[12] K. Maity and M. Maiti, "A numerical approach to a multiobjective optimal inventory control problem for deteriorating multi-items under fuzzy inflation and discounting," Computers \& Mathematics with Applications, vol. 55, no. 8, pp. 1794-1807, 2008.

[13] H. L. Yang, J. T. Teng, and M. S. Chern, "An inventory model under inflation for deteriorating items with stock-dependent consumption rate and partial backlogging shortages," International Journal of Production Economics, vol. 123, pp. 8-19, 2010.

[14] Z. Michalewicz, Genetic Algorithms + Data Structures = Evolution Programs, Springer, Berlin, Germany, 1992.

[15] M. Bessaou and P. Siarry, "A genetic algorithm with realvalue coding to optimize multimodal continuous functions," Structural and Multidisciplinary Optimization, vol. 23, no. 1, pp. 63-74, 2001.

[16] M. Last and S. Eyal, "A fuzzy-based lifetime extension of genetic algorithms," Fuzzy Sets and Systems, vol. 149, no. 1, pp. 131-147, 2005.

[17] F. Pezzella, G. Morganti, and G. Ciaschetti, "A genetic algorithm for the flexible job-shop scheduling problem," Computers and Operations Research, vol. 35, no. 10, pp. 3202-3212, 2008.

[18] D. Dubois and H. Prade, Fuzzy Sets and Systems, Theory and Applications, vol. 144, Academic Press, New York, NY, USA, 1980.

[19] R. Narmatha Banu and D. Devaraj, "Multi-objective GA with fuzzy decision making for security enhancement in power system," Applied Soft Computing, vol. 12, pp. 2756-2764, 2012.

[20] S. Kar, D. Das, and A. Roy, "A production-inventory model for a deteriorating item incorporating learning effect using genetic algorithm," Advances in Operations Research, vol. 2010, Article ID 146042, 26 pages, 2010.

[21] B. Liu and K. Iwamura, "Chance constrained programming with fuzzy parameters," Fuzzy Sets and Systems, vol. 94, no. 2, pp. 227-237, 1998.

[22] A. Roy, M. K. Maiti, S. Kar, and M. Maiti, "An inventory model for a deteriorating item with displayed stock dependent demand under fuzzy inflation and time discounting over a random planning horizon," Applied Mathematical Modelling, vol. 33, no. 2, pp. 744-759, 2009.

[23] B. Das, K. Maity, and M. Maiti, "A two warehouse supplychain model under possibility/ necessity/credibility measures," Mathematical and Computer Modelling, vol. 46, no. 3-4, pp. 398409, 2007. 


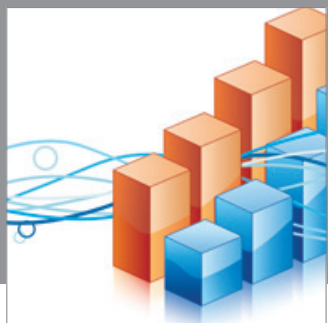

Advances in

Operations Research

mansans

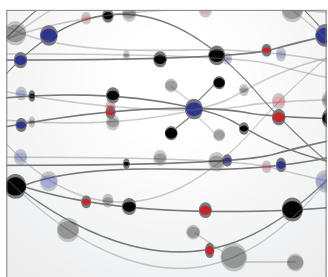

The Scientific World Journal
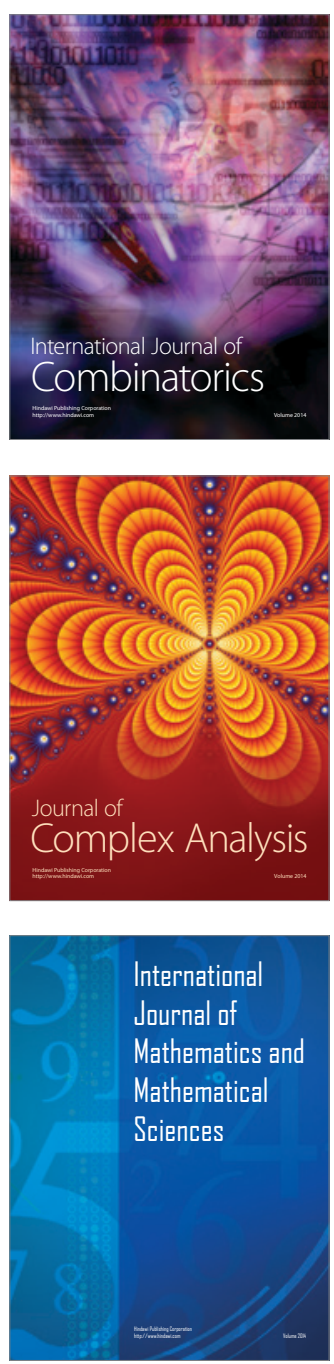
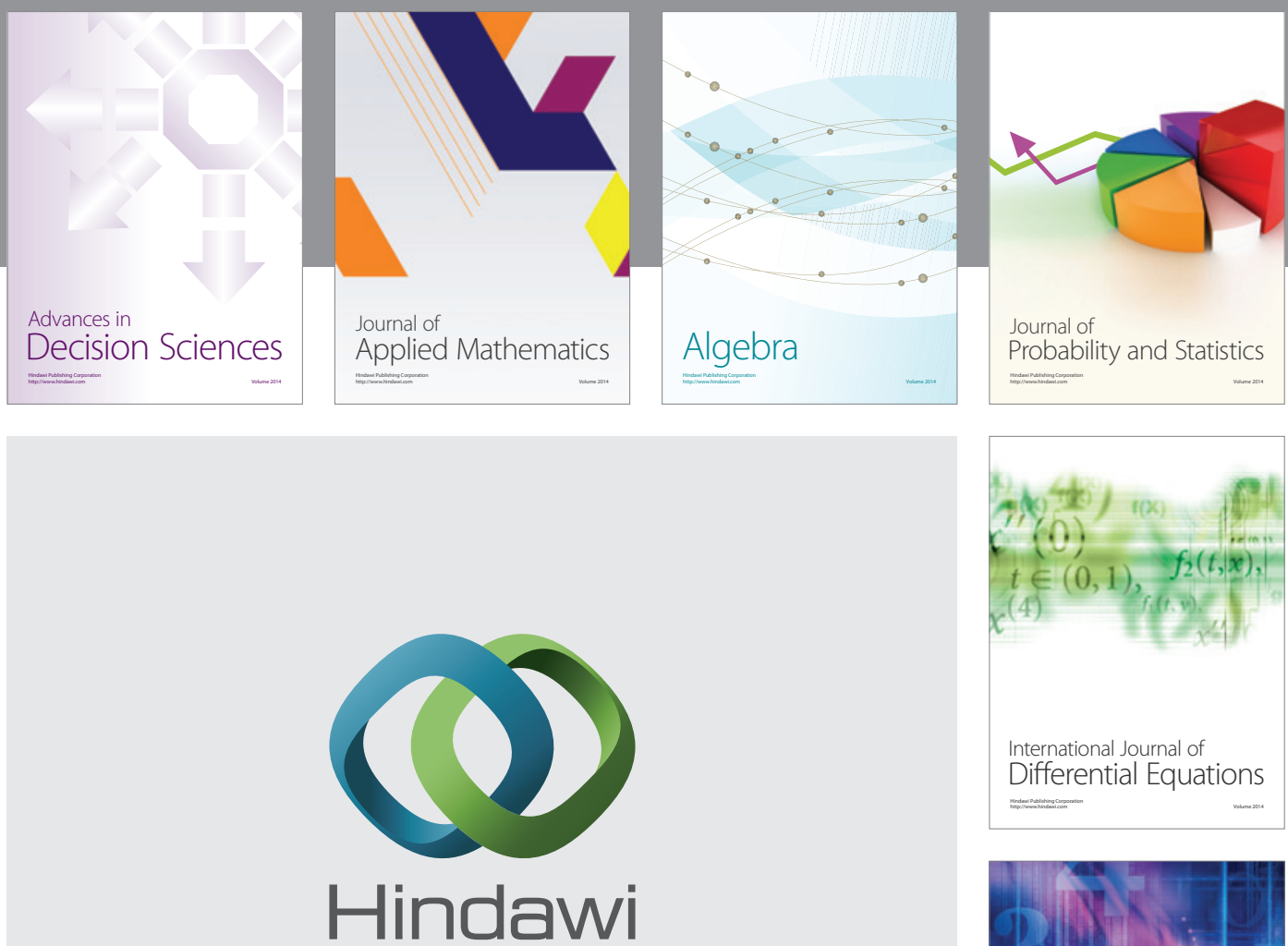

Submit your manuscripts at http://www.hindawi.com
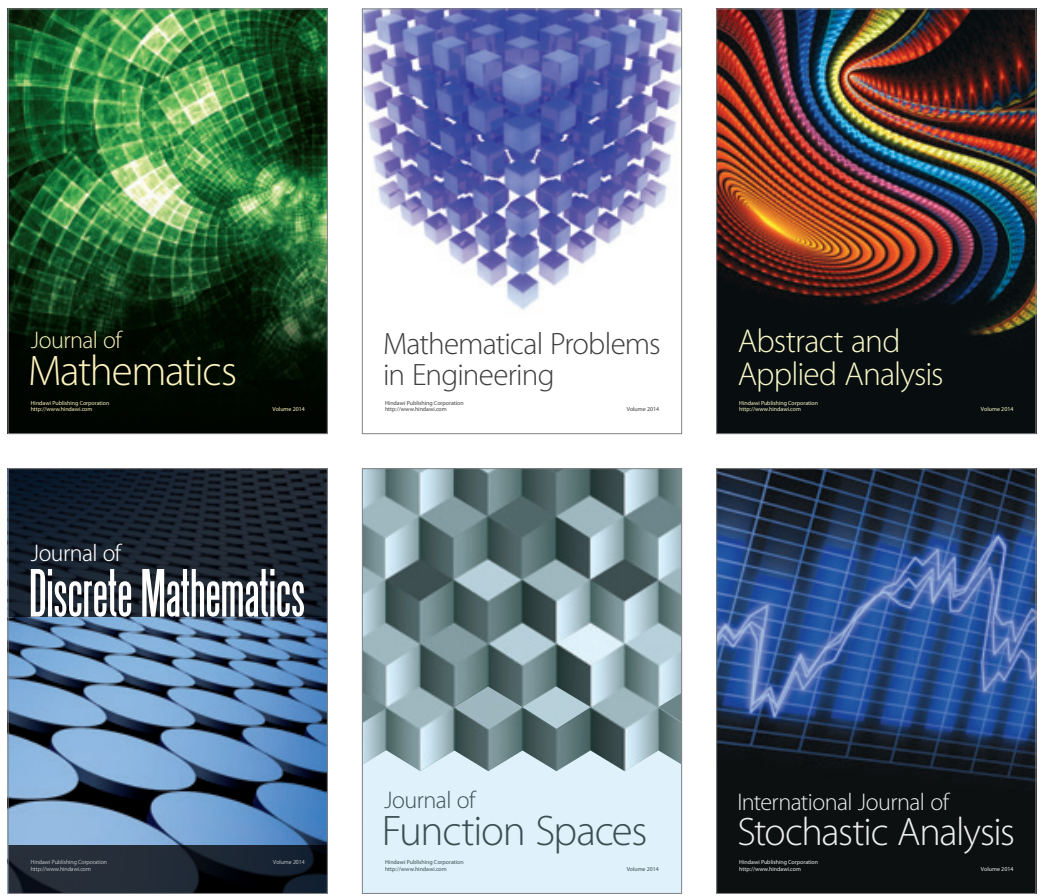

Journal of

Function Spaces

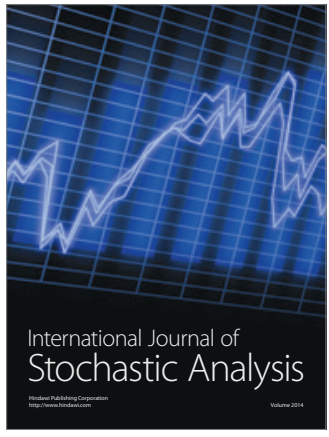

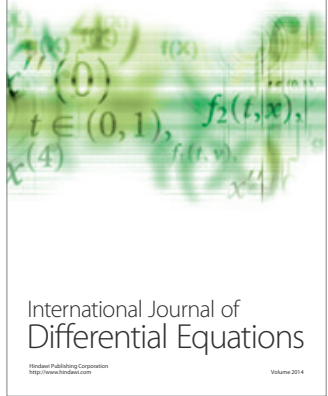
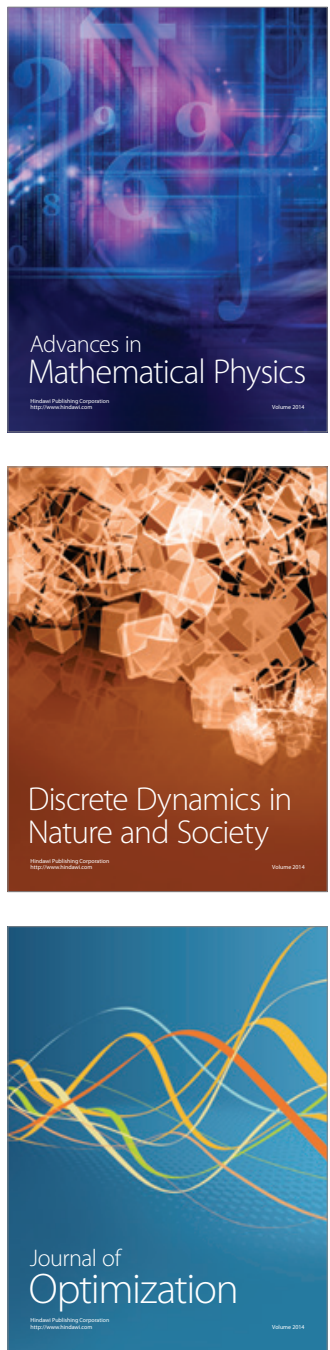